\title{
INFLUÊNCIA DA CONCENTRAÇÃO E DO PH NA DENSIDADE DE CORRENTE LIMITE DE UM SISTEMA DE MEMBRANAS DE TROCA IÔNICA POR ELETRODIÁLISE PARA SEPARAÇÃO DE COBALTO (II)*
}

\author{
Gustavo Coelho Feijoo ${ }^{1}$ \\ Tatiana Scarazzato ${ }^{2}$ \\ Denise Crocce Romano Espinosa ${ }^{3}$ \\ Jorge Alberto Soares Tenório ${ }^{4}$
}

\begin{abstract}
Resumo
Técnicas de separação por membranas têm sido consideradas alternativas para recuperação de metais valiosos. Uma destas técnicas é a eletrodiálise, em que o fenômeno da polarização por concentração causa alterações indesejáveis para o mecanismo de transporte iônico. Para evitar este problema, a densidade de corrente limite deve ser analisada. Para tanto, a influência da concentração de $\mathrm{Co}^{+2}$ e do $\mathrm{pH}$ na densidade de corrente limite foi estudada por meio de curvas corrente-voltagem. Pulsos de corrente elétrica foram aplicados entre dois eletrodos separados por membranas de troca iônica catiônica (HDX100) e aniônica (HDX200), e a diferença de potencial entre a membrana analisada foi medida em função do tempo. De acordo com os resultados, o aumento da concentração causa um aumento na densidade de corrente limite e menor efeito da polarização por concentração. Por outro lado, o aumento do $\mathrm{pH}$ causa uma queda na densidade de corrente limite devido à menor quantidade de íons $\mathrm{H}^{+}$. Os resultados indicaram também a deposição de compostos de cobalto no eletrodo de trabalho.
\end{abstract}

Palavras-chave: Eletrodiálise; Polarização por concentração; Cronopotenciometria; Técnica de separação por membranas.

\section{INFLUENCE OF CONCENTRATION AND PH ON THE LIMITING CURRENT DENSITY OF AN ELECTRODIALYSIS ION EXCHANGE MEMBRANE SYSTEM FOR COBALT (II) SEPARATION.}

\begin{abstract}
Membrane separation techniques have been considered alternatives for the recovery of valuable metals. One of these techniques is electrodialysis, in which the concentration polarization phenomenon may cause undesirable alterations in the ionic transport mechanism. To avoid this problem, the limiting current density has to be evaluated. Therefore, the influence of the $\mathrm{Co}^{+2}$ concentration and $\mathrm{pH}$ on the limiting current density was studied by means of current-voltage curves. Electrical current pulses were applied between two electrodes separated by a cation-exchange membrane (HDX100) and an anion-exchange membrane (HDX200), and the potential drop across the analyzed membrane was measured as a function of time. According to the results, the increase of cobalt concentration causes an increase on the limiting current and a smaller effect of concentration polarization. On the other hand, the $\mathrm{pH}$ increase reduces the drop on limiting current density due to the smaller amount of $\mathrm{H}^{+}$ions. The results also showed a deposition of cobalt hydroxide on the work electrode.
\end{abstract}

Keywords: Electrodialysis; Concentration polarization; Chronopotentiometry; Membrane separation techniques.

1 Engenheiro Químico, Mestrando pelo Departamento de Engenharia Química, Escola Politécnica da Universidade de São Paulo, São Paulo, São Paulo, Brasil.

2 Engenheira Mecânica, Mestre em Ciências, Doutoranda do Departamento de Engenharia Química, Escola Politécnica da Universidade de São Paulo, São Paulo, São Paulo, Brasil.

3 Engenheira Metalurgista, Doutora em Engenharia Metalúrgica, Professora Associada, Departamento de Engenharia Química, Escola Politécnica da Universidade de São Paulo, São Paulo, São Paulo, Brasil. 
4 Engenheiro Metalurgista, Doutor em Engenharia Metalúrgica, Professor Titular, Departamento de Engenharia Química, Escola Politécnica da Universidade de São Paulo, São Paulo, São Paulo, Brasil. 


\section{INTRODUÇÃO}

Diante da redução dos teores de níquel em minérios sulfetados [1-4], novos estudos têm sido realizados para extração e processamento de minérios oxidados, ou lateríticos. Apesar do seu processamento ser mais complexo, os minérios lateríticos de níquel possuem normalmente maiores teores de cobalto que podem agregar valor ao seu processamento [5]. Além disso, o tratamento de rejeitos que não apresentavam viabilidade econômica para recuperação de seus metais de interesse, atualmente se tornaram foco de estudos devido ao seu potencial econômico viabilizado pelo advento de novas tecnologias para extração e recuperação [6-9]. Soma-se a isso o interesse socioambiental quanto ao descarte de poluentes no meio ambiente $[4,6]$.

Dentre as tecnologias para recuperação de metais de resíduos líquidos e para tratamento de efluentes, a eletrodiálise tem sido estudada para diferentes aplicações [7]. Este processo permite a separação de cátions e ânions através da aplicação de uma corrente elétrica entre dois eletrodos como força motriz, separados entre si por membranas catiônicas e aniônicas. Os solutos das soluções são transportados uma combinação de mecanismos de eletromigração, difusão e convecção através de canais iônicos contidos nas membranas, purificando o solvente e propiciando a recuperação dos íons de interesse [10-13].

Dentre os metais com maior valor agregado, o cobalto e o níquel têm propriedades químicas e físicas similares e normalmente ambos estão presentes em subprodutos do processamento dos minérios, sendo sua separação um problema prático. Tecnologias tradicionais como a osmose reversa e a evaporação possuem desvantagens como a não operação em pressão e temperatura ambientes. Já a precipitação demanda mudanças de fase dos constituintes da solução. Assim, a eletrodiálise pode apresentar menor consumo energético. Além disso, as tecnologias convencionais possuem outras desvantagens em comparação com a eletrodiálise, como a necessidade de adição de mais produtos químicos durante o tratamento dos resíduos e a geração de resíduos sólidos $[9,14,15]$.

\subsection{Polarização por concentração}

Para uma corrente elétrica aplicada entre dois eletrodos num sistema de membranas de troca iônica imersas numa solução eletrolítica, tanto os cátions quanto os ânions transportam a corrente elétrica do seio da solução até as membranas. Quando os íons carregados alcançam a superfície das membranas, o transporte de cargas que antes era realizado por cátions e ânions passa a ser exercido apenas pelos cátions, no caso das membranas catiônicas, ou apenas pelos ânions, nas membranas aniônicas. Dessa forma, com o aumento da corrente elétrica, aumenta-se o gradiente de concentração de íons entre o seio da solução e a camada limite adjacente à superfície da membrana [16-18]. A escassez de íons habilitados ao transporte pela membrana ocasiona um fenômeno chamado polarização por concentração. Por isso, a determinação da densidade corrente limite é fundamental para a melhor operação do processo.

\subsection{Cronopotenciometria}

A determinação da densidade de corrente limite de um sistema de eletrodiálise pode ser realizada por cronopotenciometria. A medição do potencial de 
resposta em função do tempo é realizada por meio da aplicação de pulsos fixos de corrente elétrica entre as faces da membrana estudada $[19,20]$.

\subsection{Curvas Corrente-Voltagem (CVC)}

A partir das curvas cronopotenciométricas, curvas corrente-voltagem são construídas por meio das quais é possível identificar três regiões características $[12,17,21,22]$, conforme o exemplo apresentado na Figura 1.

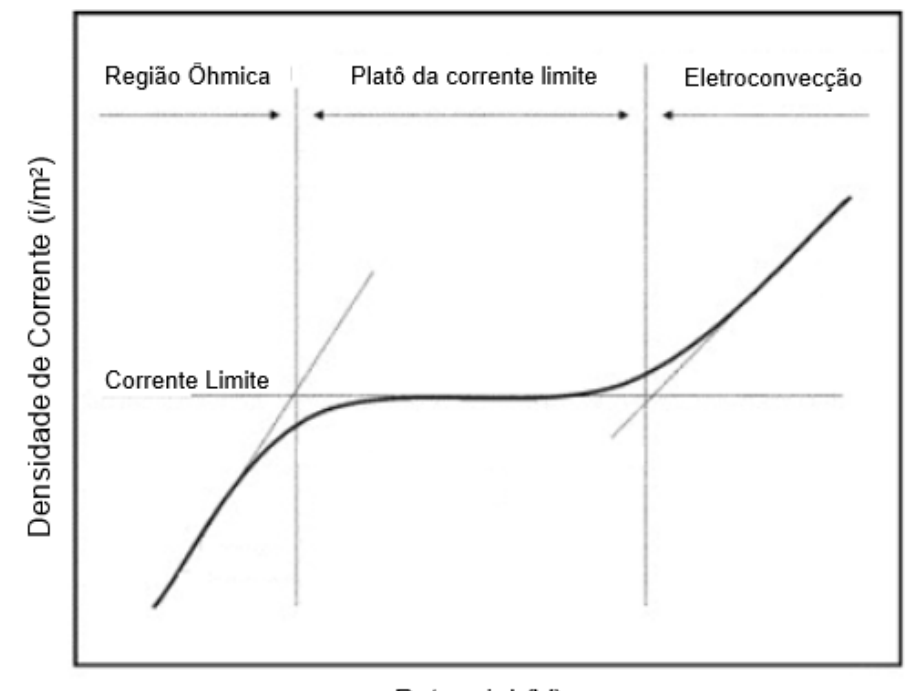

Potencial (V)

Figura 1 - CVC ou curva de polarização típica de uma membrana [17,23,24]

A região I, comumente chamada de região ôhmica, é caracterizada por um aumento linear do potencial conforme aumenta-se a corrente. A região II apresenta a zona de platô e marca a ocorrência do fenômeno da polarização por concentração. A interseção das tangentes relacionadas às regiões I e II fornecem 0 valor de corrente limite [25]. A região III apresenta novamente um aumento da corrente de acordo com o potencial aplicado devido a mecanismos como a eletroconvecção ou a hidrólise da água [12,17,21,22].

\subsection{Equação da densidade de corrente limite}

Como forma de comparação com os dados experimentais, a equação de Peer (Equação 1), pode ser utilizada para cálculo da densidade de corrente limite teórica $\left(i_{\text {lim }}\right)$ :

$$
i_{\text {lim }}=\frac{F \cdot C_{0} \cdot D \cdot z_{j}}{\delta \cdot\left(t_{j}^{m}-t_{j}^{s}\right)}
$$

Nesta equação, $F$ é a constante de Faraday, $C_{0}$ é a concentração de sal no seio da solução, $D$ é o coeficiente de difusão do sal na solução, $z_{j}$ a carga do contraíon, $\delta$ a espessura da camada limite de difusão, $t_{j}^{m}$ e $t_{j}^{s}$ os números de transporte do contra-íon na membrana e na solução, respectivamente. 


\subsection{Objetivos}

Diante do exposto, o presente trabalho tem como objetivo comparar o efeito da variação da concentração de cátions de cobalto e do pH numa membrana catiônica heterogênea, pela determinação da densidade de corrente limite de através de curvas corrente-voltagem (CVC).

\section{MATERIAIS E MÉTODOS}

Soluções de $\mathrm{CoSO}_{4} .7 \mathrm{H}_{2} \mathrm{O}$ em grau analítico foram adicionadas em um sistema de três compartimentos construído em acrílico transparente, com $130 \mathrm{~mL}$ cada. O compartimento central foi separado dos externos por uma membrana heterogênea catiônica (HDX 100) de um lado, e por uma membrana heterogênea aniônica (HDX 200) do outro. As membranas foram fornecidas pela empresa Hidrodex®. As especificações das membranas empregadas estão presentes na Tabela 1. Para o eletrodo de trabalho e para o contra-eletrodo, foram utilizadas ponteiras de grafite. O potencial da membrana catiônica foi medido em temperatura ambiente por dois eletrodos de referência de $\mathrm{Ag} / \mathrm{AgCl}$ imersos em capilares de Luggin contendo a mesma solução, e sem agitação, conforme mostra a Figura 2. A área das membranas em estudo foi de $3,14 \mathrm{~cm} 2$. As medições de $\mathrm{pH}$ e condutividade foram realizadas com o medidor multiparâmetros modelo Lovibond Sensodirect 150. 
Tabela 1 - Propriedades das membranas HDX catiônica (100) e aniônica (200).

\begin{tabular}{|c|c|c|c|}
\hline Propriedade & HDX 100 & HDX 200 & Unidade \\
\hline Grupo lônico Fixado & $-\mathrm{SO}_{3}^{-}$ & $-\mathrm{NR}_{3}^{+}$ & - \\
\hline Contenção de Água & $35-50$ & $30-45$ & $\%$ \\
\hline Capacidade de Troca iônica & $\geq 2,0$ & $\geq 1,8$ & $\begin{array}{l}\text { Mol. } \mathrm{Kg}^{-1} \\
\text { (seca) }\end{array}$ \\
\hline $\begin{array}{l}\text { Resistência Superficial }(0,1 \\
\text { mol de } \mathrm{NaCl})\end{array}$ & $\leq 20$ & $\leq 20$ & Ohm.cm² \\
\hline $\begin{array}{l}\text { Penetração Seletiva }(0,1 \\
\text { mol KCl / 0,2 mol KCl) }\end{array}$ & $\geq 90$ & $\geq 89$ & $\%$ \\
\hline Resistência de ruptura & $\geq 0,6$ & $\geq 0,6$ & $\mathrm{MPa}$ \\
\hline Permeabilidade da água & $\begin{array}{c}\leq 0,1 \text { (abaixo de } \\
0,2 \mathrm{Mpa})\end{array}$ & $\begin{array}{c}\leq 0,2 \text { (abaixo de } \\
0,035 \mathrm{Mpa})\end{array}$ & mL.h.cm ${ }^{-2}$ \\
\hline
\end{tabular}

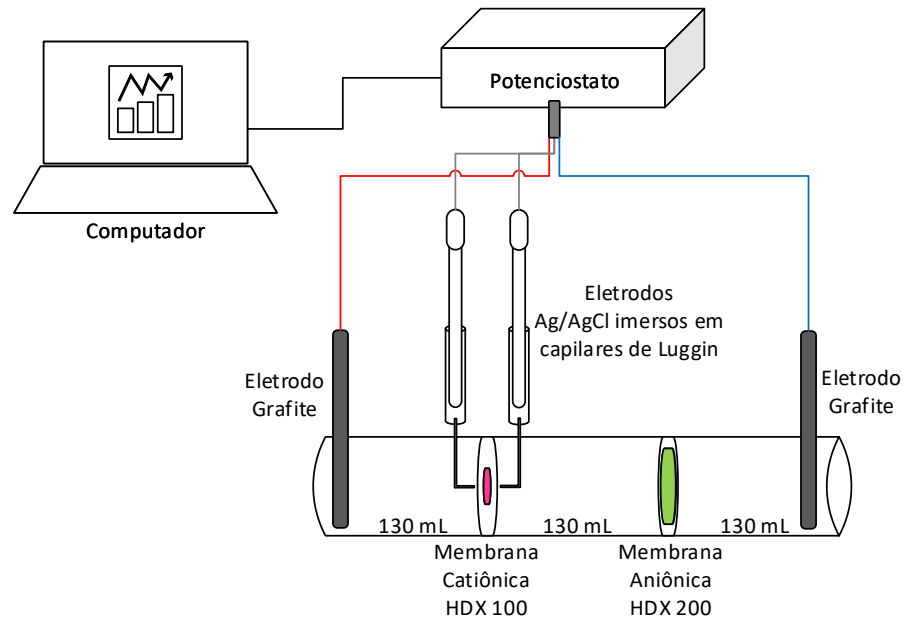

Figura 2 - Sistema para estudo da densidade de corrente limite.

\subsection{Avaliação da concentração}

Para estudo da influência da concentração na densidade de corrente limite, foram preparadas soluções de sulfato de cobalto hepta-hidratado $\mathrm{CoSO}_{4} .7 \mathrm{H}_{2} \mathrm{O}$ em três concentrações: $0,1 \mathrm{~g} / \mathrm{L}, 0,3 \mathrm{~g} / \mathrm{L}$ e $0,5 \mathrm{~g} / \mathrm{L}$ de $\mathrm{Co}^{+2}$. $\mathrm{O} \mathrm{pH}$ inicial das soluções foi ajustado para 4 com $\mathrm{H}_{2} \mathrm{SO}_{4} 0,1 \mathrm{M}$. Água deionizada Milli-Q foi utilizada para preparar as soluções.

\subsection{Avaliação do $\mathrm{pH}$}

Para estudo da influência do $\mathrm{pH}$ na densidade de corrente limite, foram preparadas soluções de sulfato de cobalto hepta-hidratado $\mathrm{CoSO}_{4} .7 \mathrm{H}_{2} \mathrm{O}$ ajustando $\mathrm{O}$ $\mathrm{pH}$ inicial com $\mathrm{H}_{2} \mathrm{SO}_{4}$ 0,1M em três valores: 2,5; 3,5 e 4,5. A concentração das soluções foi de $0,3 \mathrm{~g} / \mathrm{L}$ de $\mathrm{Co}^{+2}$. Água deionizada Milli- $\mathrm{Q}$ foi utilizada para preparar as soluções.

\subsection{Ensaios}

Para construção das curvas cronopotenciométricas, foram aplicados pulsos de corrente elétrica por $300 \mathrm{~s}$, de forma que o estado estacionário fosse atingido. Após interrompido cada pulso de corrente, um tempo de $100 \mathrm{~s}$ foi estipulado para relaxamento do sistema. Os valores de potencial para cada corrente aplicada foram 
coletados no momento imediatamente antes da interrupção da corrente. Um potenciostato modelo Biologic SP-50 foi utilizado para aplicação dos valores de corrente. Os ensaios realizados são mostrados na Tabela 2.

Tabela 2 - Concentrações de $\mathrm{Co}^{+2} \mathrm{e}$ valores de $\mathrm{pH}$ avaliados nos ensaios.

\begin{tabular}{cccc}
\hline $\begin{array}{c}\text { Parâmetro } \\
\text { Variado }\end{array}$ & Ensaio & $\mathbf{C o}^{+2} \mathbf{( g / L )}$ & $\mathbf{p H}$ \\
\hline \multirow{2}{*}{ Concentração } & $\mathrm{A}$ & 0,1 & 4,0 \\
& $\mathrm{~B}$ & 0,3 & 4,0 \\
& $\mathrm{C}$ & 0,5 & 4,0 \\
$\mathrm{pH}$ & $\mathrm{D}$ & 0,3 & \\
& $\mathrm{E}$ & 0,3 & 3,5 \\
& $\mathrm{~F}$ & 0,3 & 4,5 \\
\hline
\end{tabular}

\section{RESULTADOS E DISCUSSÃO}

Pelas curvas corrente-voltagem obtidas, foi possível identificar três regiões bem definidas, de acordo com a literatura $[16,17,26]$. A corrente limite para cada sistema foi determinada pela intersecção das regiões I e II [25], e os valores obtidos são mostrados na Tabela 3. 
Tabela 3 - Densidades de corrente limite, espessuras da camada de difusão e números de transporte para cada ensaio realizado.

\begin{tabular}{|c|c|c|c|c|c|c|c|}
\hline Ensaio & $\begin{array}{l}\mathrm{Co}^{+2} \\
(\mathrm{~g} / \mathrm{L})\end{array}$ & $\mathrm{pH}$ & $\begin{array}{l}\lim _{\lim } \text { exp. } \\
\left(\mathrm{A} / \mathrm{m}^{2}\right)\end{array}$ & $\begin{array}{l}\text { lim teo. } \\
\left(A / m^{2}\right)\end{array}$ & $\delta(\mu \mathrm{m})$ & $t_{j}{ }^{s}$ & $t_{j}{ }^{m}$ \\
\hline$A$ & 0,1 & 4,0 & 1,5 & 1,2 & 579 & 0,39 & 0,81 \\
\hline$B$ & 0,3 & 4,0 & 5,5 & 4,8 & 440 & 0,39 & 0,80 \\
\hline C & 0,5 & 4,0 & 9,3 & 8,5 & 387 & 0,39 & 0,83 \\
\hline D & 0,3 & 2,5 & 17,2 & 14,5 & 440 & 0,39 & 0,53 \\
\hline$E$ & 0,3 & 3,5 & 5,6 & 5,1 & 440 & 0,39 & 0,77 \\
\hline$F$ & 0,3 & 4,5 & 5,0 & 4,4 & 440 & 0,39 & 0,84 \\
\hline
\end{tabular}

\subsection{Avaliação da concentração}

Observou-se pelas das curvas corrente-potencial do ensaios $A, B$ e $C$ apresentadas na Figura 3, que a densidade de corrente limite aumenta com o aumento da concentração de íons, em conformidade com os resultados obtidos por Krol [16], Káňavová [24], Herraiz-Cardona [27] e Barros [22]. Este comportamento é explicado pela maior quantidade de íons que carregam a corrente elétrica. Consequentemente, a concentração por polarização devido à escassez de íons ocorrerá em densidades de corrente maiores.

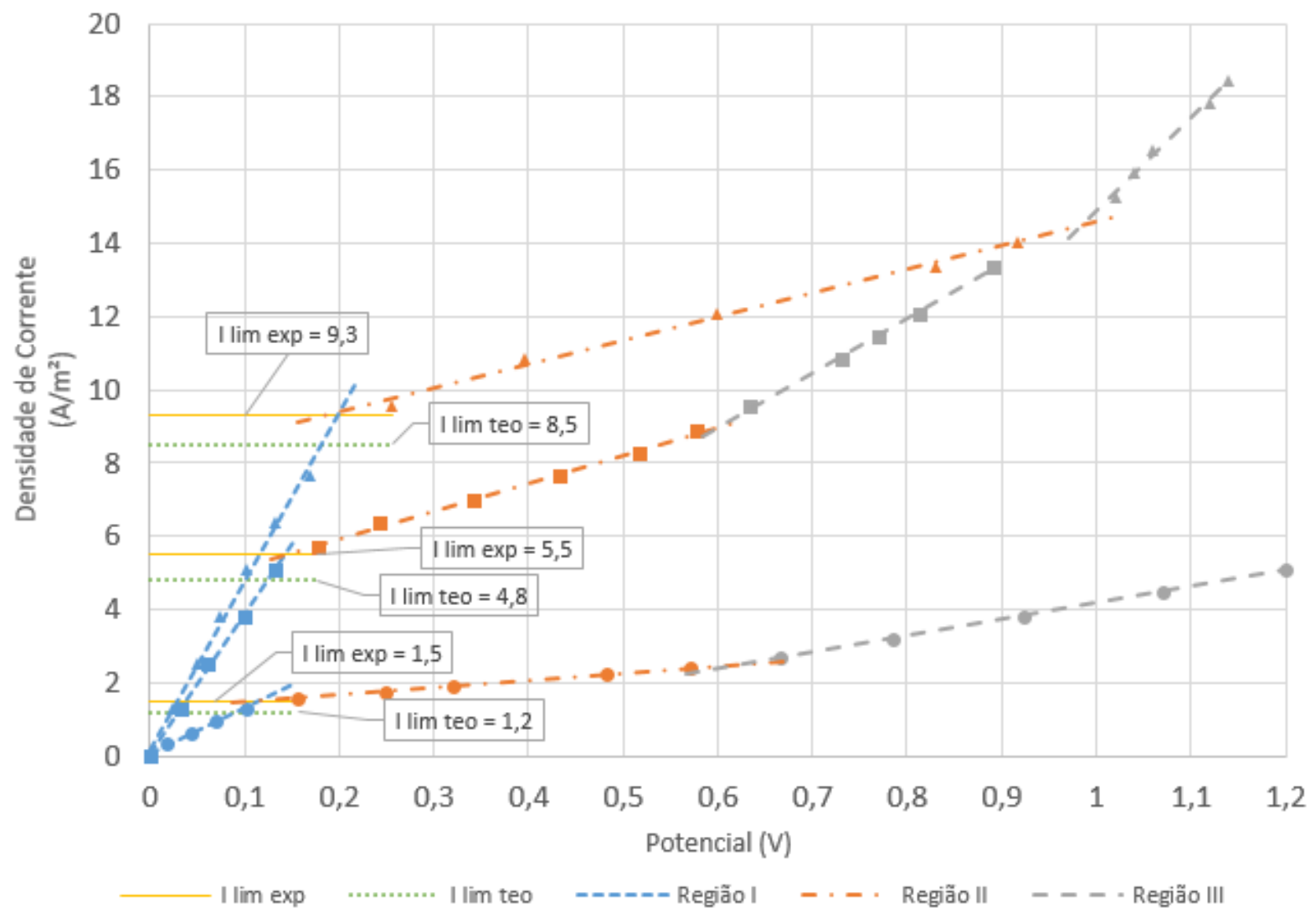

Figura 3 - Curvas corrente-potencial para $0,1 \mathrm{~g} / \mathrm{L}$ de $\mathrm{Co}^{+2}$ e $\mathrm{pH}=4(\mathrm{~A}, \bullet) ; 0,3 \mathrm{~g} / \mathrm{L}$ de $\mathrm{Co}^{+2}$ e $\mathrm{pH}=4(\mathrm{~B}, \mathbf{\square})$; $0,5 \mathrm{~g} / \mathrm{L}$ de $\mathrm{Co}^{+2}$ e $\mathrm{pH}=4(\mathrm{C}, \mathbf{\Lambda})$.

Pela equação 1 de Peer, também pode-se destacar a contribuição da espessura da camada limite (ס) para a polarização por concentração. Quanto menor é a espessura, maior será a corrente limite do sistema. Isto pôde ser confirmado pelos valores obtidos nos ensaios A, B e C, apresentados na Tabela 3. A diminuição 
da camada limite pode ser alcançada pelo aumento da velocidade de fluxo e consequente elevação da turbulência no sistema [28].

\subsection{Avaliação do pH}

Com relação à variação do $\mathrm{pH}$ para uma concentração constante de $\mathrm{Co}^{+2}$ (Figura 4), observou-se que, quanto maior o pH, menor é a densidade de corrente limite. Isto é explicado da mesma forma que a concentração de íons de sais, em que quanto maior a concentração hidrogeniônica na solução, maior a disponibilidade de íons $\mathrm{H}^{+}$para transporte da corrente pelas membranas catiônicas. Os resultados corroboram com o encontrado por Barros [22], para uma solução contendo íons de $\mathrm{Cu}^{+2}$. No entanto, deve-se salientar que quanto menor os valores de $\mathrm{pH}$, maior a competição por sítios ativos na membrana entre os cátions de cobalto e os cátions $\mathrm{H}^{+}$, e consequentemente menor a eficiência da separação dos cátions de interesse. Isto foi comprovado pelos valores obtidos nos ensaios D, E e F, apresentados na Tabela 3, em que a diminuição do pH ocasionou uma diminuição do número de transporte na membrana $\left(\mathrm{t}_{\mathrm{j}}^{\mathrm{m}}\right)$ para os cátions de $\mathrm{Co}^{+2}$.

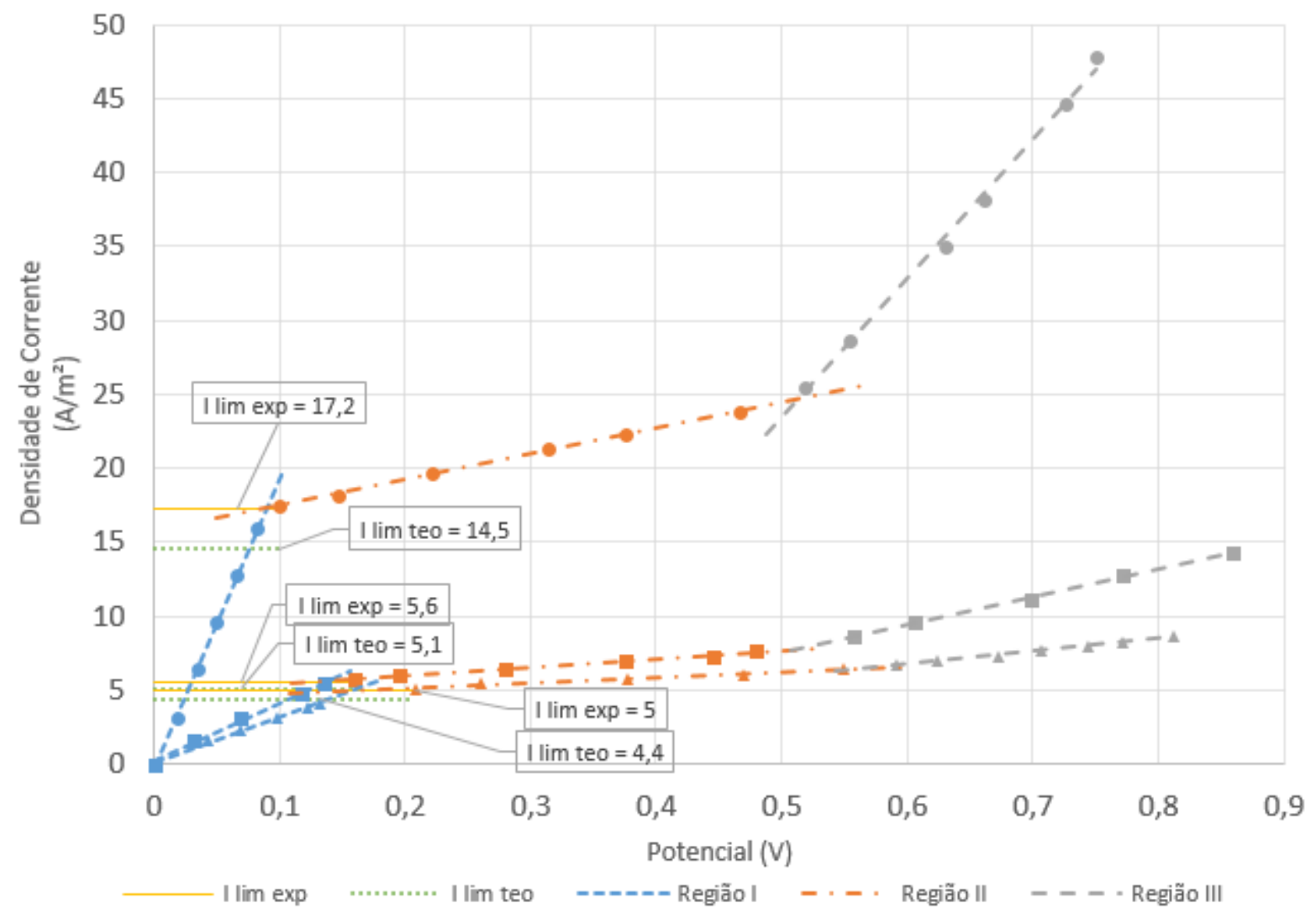

Figura 4 - Curvas corrente-potencial para $0,3 \mathrm{~g} / \mathrm{L}$ de $\mathrm{Co}^{+2}$ e $\mathrm{pH}=2,5(\mathrm{D}, \bullet) ; 0,3 \mathrm{~g} / \mathrm{L}$ de $\mathrm{Co}^{+2}$ e $\mathrm{pH}=3,5$ $(\mathrm{E}, \boldsymbol{\square}) ; 0,3 \mathrm{~g} / \mathrm{L}$ de $\mathrm{Co}^{+2}$ e $\mathrm{pH}=4,5(\mathrm{~F}, \mathbf{\Lambda})$.

Além disso, observou-se que no ensaio com $\mathrm{pH}$ inicial de 3,5 e mais acentuadamente no de $\mathrm{pH} 4,5$ (ensaios $\mathrm{E}$ e $\mathrm{F}$ respectivamente), houve deposição de hidróxido de cobalto na interface do eletrodo de trabalho (cátodo), conforme mostra a Figura 5. 


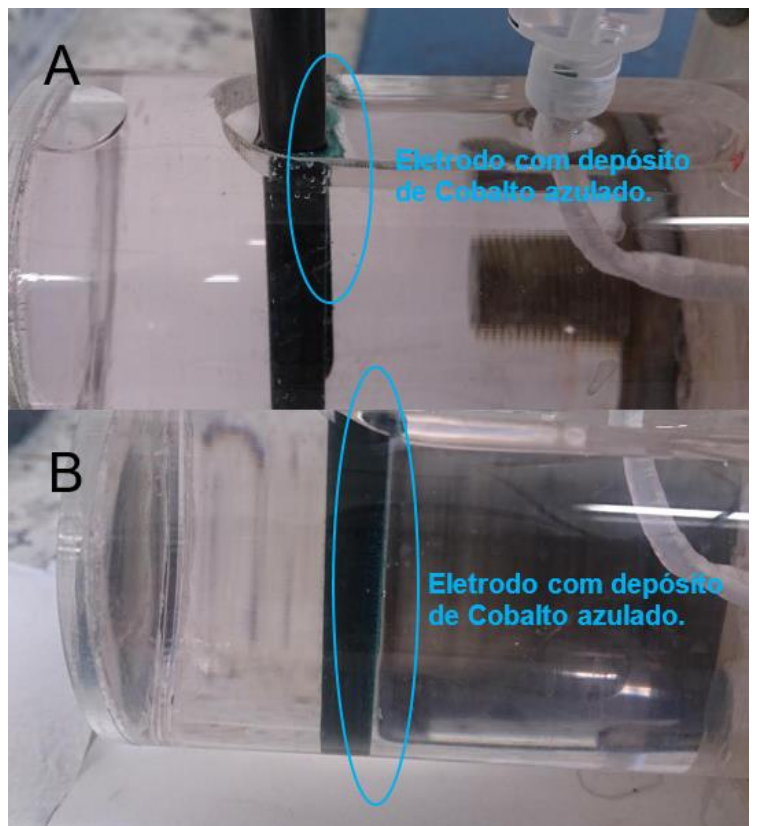

Figura 5 - Depósito de cobalto após ensaio com pH 3,5 (A); e pH 4,5 (B)

No entanto, o gráfico Concentração-pH obtido pelo software Hydra-Medusa (Figura 6) mostra que a formação de precipitado cristalino de $\mathrm{Co}(\mathrm{OH})_{2}$ ocorre somente em valores de $\mathrm{pH}$ acima de 7,5.

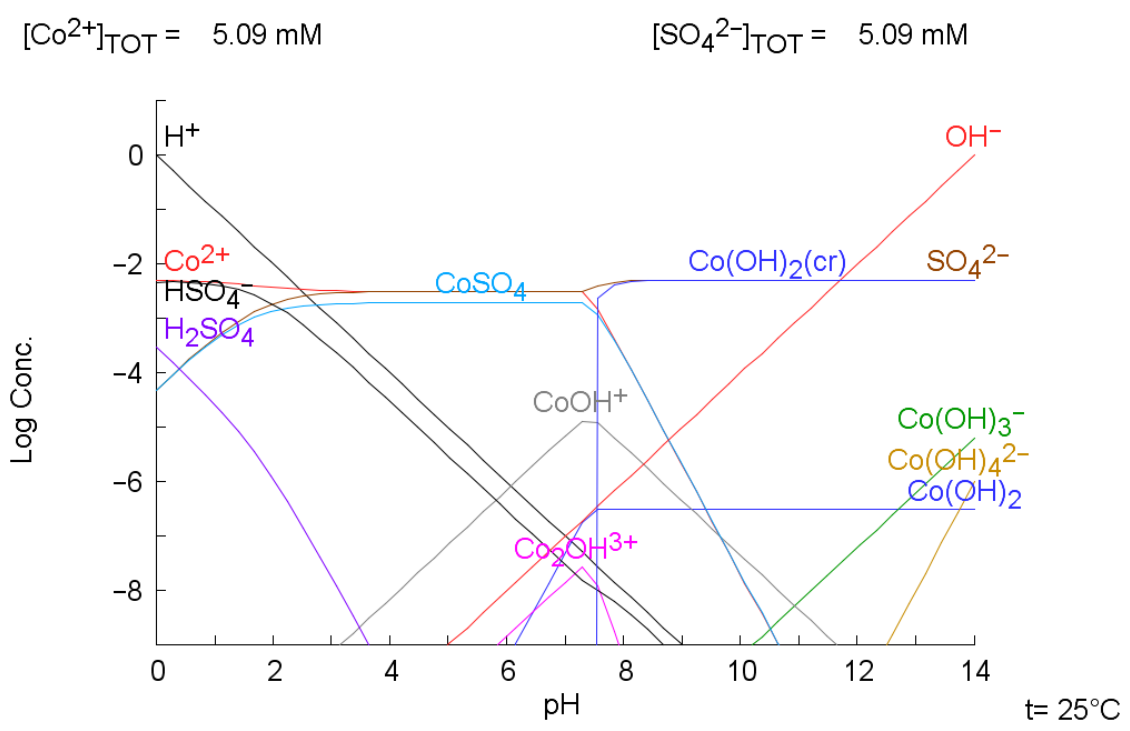

Figura 6 - Log da concentração molar de $\mathrm{Co}^{+2}(0,3 \mathrm{~g} / \mathrm{L})$ em função do $\mathrm{pH}$

Desse modo, a deposição de hidróxido de cobalto pode ser explicada pela densidade de corrente acima do valor limite, quando se inicia a dissociação da água no cátodo representada pela equação 2 , e consequente formação do gás hidrogênio (Equação 3) [16,29]:

$$
\begin{gathered}
2 \mathrm{H}_{2} \mathrm{O} \rightarrow 2 \mathrm{H}^{+}+2 \mathrm{OH}^{-} \\
2 \mathrm{H}^{+}+2 \mathrm{e}^{-} \rightarrow \mathrm{H}_{2}
\end{gathered}
$$


Quanto maior o consumo de íons $\mathrm{H}^{+}$pela formação de $\mathrm{H}_{2}$ durante a hidrólise da água, maior é o valor do $\mathrm{pH}$ devido à elevada disponibilidade de ânions $\mathrm{OH}^{-}$na interface do cátodo. Assim, quanto menor o valor do pH inicial, menor a propensão do sistema de atingir $\mathrm{pH}$ 7,5 pela dissociação da água na interface do cátodo, e consequentemente menor será a formação do precipitado $\mathrm{Co}(\mathrm{OH})_{2}$.

\section{CONCLUSÃO}

Os resultados comprovaram que para a membrana analisada, quanto maior a concentração de íons na solução, maior a disponibilidade de íons para transporte da corrente elétrica, dificultando o fenômeno da polarização por concentração pela escassez de íons na camada limite das membranas. Consequentemente, a densidade de corrente limite é proporcional ao aumento da concentração de íons.

No entanto, quanto maior o valor do $\mathrm{pH}$, menor é a densidade de corrente limite, dado que a concentração de cátions $\mathrm{H}^{+}$diminui na camada limite, facilitando a ocorrência do fenômeno da polarização por concentração. Observou-se também a influência do pH na deposição de hidróxido de cobalto no eletrodo de trabalho, que pode diminuir a eficiência da eletrodiálise.

\section{Agradecimentos}

Os autores agradecem à Coordenação de Aperfeiçoamento de Pessoal de Nível Superior (CAPES) e ao Conselho Nacional de Desenvolvimento Científico e Tecnológico (CNPq) pelas bolsas de mestrado e doutorado concedidas.

À Fundação de Amparo à Pesquisa do Estado de São Paulo (FAPESP) pelo suporte financeiro através do projeto de pesquisa 2012/51871-9.

\section{REFERÊNCIAS}

1 Farrokhpay S, Filippov L. Challenges in processing nickel laterite ores by flotation. Int $\mathrm{J}$ Miner Process. 2016;151:59-67.

2 Kursunoglu S, Kaya M. Atmospheric pressure acid leaching of Caldag lateritic nickel ore. Int J Miner Process. 2016;150:1-8..

3 Zhang Y-L, Wang C-Y, Yang Y-Q, Yin F, Ma B-Z. Pressure nitric acid leaching of alkalipretreated low-grade limonitic laterite. Rare Met. 2015;34:64-70.

4 Behera SK, Floribert Mulaba-Bafubiandi A. Advances in microbial leaching processes for nickel extraction from lateritic minerals - A review. Korean J Chem Eng. 2015;32(8):1447-54.

5 Norgate T, Jahanshahi S. Assessing the energy and greenhouse gas footprints of nickel laterite processing. Miner Eng. 2011;24(7):698-707.

6 Veselská V, Majzlan J. Environmental impact and potential utilization of historical CuFe-Co slags. Environ Sci Pollut Res. 2016;23(8):7308-23.

7 Fu F, Wang Q. Removal of heavy metal ions from wastewaters: A review. J Environ Manage. 2011;92(3):407-18.

8 Mahmoud A, Hoadley AFA. An evaluation of a hybrid ion exchange electrodialysis process in the recovery of heavy metals from simulated dilute industrial wastewater. Water Res. 2012;46(10):3364-76.

9 Frioui S, Oumeddour R, Lacour S. Highly selective extraction of metal ions from dilute solutions by hybrid electrodialysis technology. Sep Purif Technol. 2017;174:264-74.

10 Strathmann H. Ion-Exchange Membrane Separation Processes. Elsevier; 2004. 1-348 p. 
11 Benvenuti T, Krapf RS, Rodrigues MAS, Bernardes AM, Zoppas-Ferreira J. Recovery of nickel and water from nickel electroplating wastewater by electrodialysis. Sep Purif Technol. 2014;129:106-12.

12 Scarazzato T, Moisés VBR, Panossian Z, Tenório JAS, Espinosa DCR. Aplicação Da Cronopotenciometria Para Avaliação Do Transporte De Íons De Rejeito De Eletrodeposição De Cobre Através De Uma Membrana Seletiva. In: Associação Brasileira de Metalurgia, Materiais e Mineração. (ABM). 2015.

13 Deng D, Aouad W, Braff WA, Schlumpberger S, Suss ME, Bazant MZ. Water purification by shock electrodialysis: Deionization, filtration, separation, and disinfection. Desalination. 2015;357:77-83.

14 Strathmann H. Membranes and Membrane Separation Processes. In: Ullmann's Encyclopedia of Industrial Chemistry. Wiley-VCH Verlag GmbH \& Co. KGaA, Weinheim; 2012. p. 413-54.

15 Sadyrbaeva TZ. Separation of cobalt(II) from nickel(II) by a hybrid liquid membraneelectrodialysis process using anion exchange carriers. Desalination. 2015;365:167-75.

$16 \mathrm{Krol} \mathrm{JJ}$, Wessling M, Strathmann $\mathrm{H}$. Concentration polarization with monopolar ion exchange membranes: current-voltage curves and water dissociation. J Memb Sci. 1999;162(1):145-54.

17 Marder L, Ortega Navarro EM, Perez-Herranz V, Bernardes AM, Ferreira JZ. Evaluation of transition metals transport properties through a cation exchange membrane by chronopotentiometry. J Memb Sci. 2006;284(1-2):267-75.

18 Martí-Calatayud MC, Buzzi DC, García-Gabaldón M, Bernardes AM, Tenório JAS, Pérez-Herranz V. Ion transport through homogeneous and heterogeneous ionexchange membranes in single salt and multicomponent electrolyte solutions. J Memb Sci. 2014;466:45-57.

19 Krol JJ, Wessling M, Strathmann H. Chronopotentiometry and overlimiting ion transport through monopolar ion exchange membranes. J Memb Sci. 1999;162(1-2):155-64.

20 Pismenskaia N, Sistat P, Huguet P, Nikonenko V, Pourcelly G. Chronopotentiometry applied to the study of ion transfer through anion exchange membranes. J Memb Sci. 2004;228(1):65-76.

21 Barragán V, Ruíz-Bauzá C. Current-Voltage Curves for lon-Exchange Membranes: A Method for Determining the Limiting Current Density. J Colloid Interface Sci. 1998;205(2):365-73.

22 Barros KS, Scarazzato T, Tenório JAS, Espinosa DCR. Determinação Da Corrente Limite Através Da Construção De Curvas Corrente-Voltagem Para O Tratamento De Efluente Proveniente Da Galvanoplastia Contendo Cobre. In: Congresso Brasileiro de Engenharia e Ciência dos Materiais (CBECiMat). 2016.

23 Scarazzato T. Tratamento de efluente contendo HEDP por eletrodiálise [tese de mestrado]. University of São Paulo. 2013.

24 Káňavová N, Machuča L, Tvrzník D. Determination of limiting current density for different electrodialysis modules. Chem Pap. 2013

25 Scarazzato T, Buzzi DC, Bernardes AM, Romano Espinosa DC. Treatment of wastewaters from cyanide-free plating process by electrodialysis. J Clean Prod. 2015;91:241-50.

26 Tanaka Y. Concentration polarization in ion-exchange membrane electrodialysis-the events arising in a flowing solution in a desalting cell. J Memb Sci. 2003;216(1):149-64.

27 Herraiz-Cardona I, Ortega E, Pérez-Herranz V. Evaluation of the Zn2+ transport properties through a cation-exchange membrane by chronopotentiometry. J Colloid Interface Sci. 2010;341(2):380-5.

28 Baker RW. Membrane Technology and Applications. Membrane Technology and Applications. 2004. 538 p.

29 Tanaka Y. Water dissociation in ion-exchange membrane electrodialysis. J Memb Sci. 2002;203(1):227-44. 